\title{
The cascade equivalent $A-H$-circuit of the salient-pole generator on the base of the anisotropic model
}

\author{
Alexey Blanc \\ Department of Theoretical Foundation of Electrical Engineering, Novosibirsk State Technical University, Russia
}

\begin{tabular}{l}
\hline \hline Article Info \\
\hline Article history: \\
Received Feb 17, 2020 \\
Revised Jun 18, 2020 \\
Accepted Jun 30, 2020 \\
\hline
\end{tabular}

\section{Keywords:}

Cascade equivalent circuits

Electric machines

Salient-pole electric machines

Synchronous electric generators

\begin{abstract}
In the scientific studies of the electric machines, cylindrical and Cartesian laminated models have long proved their operability when it is necessary to transform the general solutions of the partial differential equations into fourterminal network equations and to create the cascade equivalent circuits of the electric machines. In the case of the salient-pole machines, piecewise continuous Sturm-Liouville eigenfunctions are usually used as the general solution. Unfortunately, we cannot create the cascade equivalent circuit of the salient-pole electric machine when (to ensure both the uniqueness and the accuracy of the solution) the field is modeled with many piecewise continuous Sturm-Liouville eigenfunctions (in the zone of poles) and many sinusoidal functions (in the air gap). Nevertheless, the author developed the approximate method for modeling the magnetic field of the salient-pole electric machines when only one piecewise continuous Sturm-Liouville eigenfunction is used and many sinusoidal functions. In this case, it becomes possible to transform the general solution of the partial differential equation into four-terminal network equations and to create the cascade equivalent circuit of the salient-pole electric machine. In this paper, the cascade equivalent $A$ - $H$-circuit of the synchronous salient-pole machine is considered (without using piecewise continuous Sturm-Liouville eigenfunctions but also without properties averaging).
\end{abstract}

This is an open access article under the CC BY-SA license.

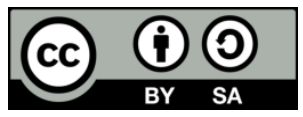

Corresponding Author:

Alexey Blanc

Department of Theoretical Foundation of Electrical Engineering

Novosibirsk State Technical University

20 Marx avenue, Novosibirsk, 630073 Russia

Email: alblances@yandex.ru

\section{INTRODUCTION}

In the second half of the twentieth century, many papers were published [1-7] that examined the problem of modeling the electromagnetic field of electric machines based on the laminated models. Each layer of the laminated model corresponded to some zone of the machine (air gaps, teeth, slots, the yokes of the stator and the rotor). The general solution of the partial differential equation had to be found in each layer of the laminated model and integration constants had to be defined (according to the boundary conditions).

Unfortunately, the calculations were so complex, and the results were so cumbersome that the laminated models did not receive any practical development in the future. However, it became apparent that the solutions of the partial differential equations that were obtained for the laminated models could be reduced into the four-terminal network equations known in the circuit theory. If now the electric intensity is considered equivalent to the voltage and the magnetic intensity is considered equivalent to the current, it is possible to create the equivalent circuit of the four-terminal network equations (the so-called $E$ - $H$-cascade equivalent circuit). If the vector magnetic potential is considered equivalent to the voltage and the magnetic 
intensity is considered equivalent to the current, we have the $A$ - $H$-cascade equivalent circuit. The cascade equivalent circuits were created for the different types of cylindrical rotor machine [8-20], as well as for electric technological installations [21-27].

In the case of the salient-pole machines, in which alternating poles and air spaces between poles cannot be considered as the homogeneous zone, piecewise continuous Sturm-Liouville eigenfunctions are usually used as the general solution of the partial differential equation [28-30]. Unfortunately, we cannot create the cascade equivalent circuit of the salient-pole electric machine when (to ensure both the uniqueness and the accuracy of the solution) the field is modeled with many piecewise continuous Sturm-Liouville eigenfunctions (in the zone of alternating poles and air spaces between poles) and many sinusoidal functions (in the air gap). Nevertheless, the author developed the approximate method for modeling the magnetic field of the salient-pole electric machines when only one piecewise continuous Sturm-Liouville eigenfunction is used (in the zone of poles) and many sinusoidal functions (in the air gap) [31, 32]. In this case, it becomes possible to transform the general solution of the partial differential equation into four-terminal network equations and to create the cascade equivalent circuit of the salient-pole electric machine [33-36]. This paper deals with the same subject as [36]. However, as opposed to [36], the $A$ - $H$-circuit of the electric machine is created without using piecewise continuous Sturm-Liouville eigenfunctions (but also without properties averaging).

\section{THE EQUIVALENT $A$ - $H$-CIRCUIT OF THE ROTOR}

The object of the study is the synchronous generator with the salient-pole rotor (that is also the inductor). Both the rotor yoke and the stator yoke are perfect ferromagnetic, their magnetic permeability is infinite. The magnetic permeability of poles is constant and finite. Poles and air spaces between poles are bounded by coordinate surfaces of the cylindrical coordinate system as shown in Figure 1.

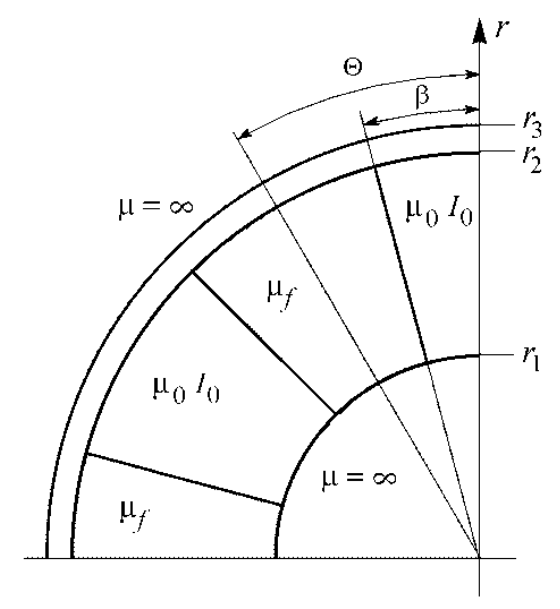

Figure 1. The salient-pole synchronous generator

The current $I_{0}$ creates the exciting field. Let us substitute equivalent current sheets (that will be located on the rotor yoke between poles) for the exciting current. Fourier's expansion turns the current sheet into

$$
H_{0}(\alpha)=\sum_{k=1,3,5}^{\infty} \frac{I_{0} \sin k p \beta}{r_{1} k p \Theta \beta} \cos k p \alpha
$$

where $2 p$ is the number of poles.

It is known that in case of both the exciting field and the longitudinal armature reaction, field lines are directed generally parallel to the $r$-axis within the poles and generally perpendicular to the $r$-axis within the air spaces between poles. Therefore, we can substitute the anisotropic zone for the poles and the air spaces where the components of the magnetic permeability must be 


$$
\begin{aligned}
& \mu_{r}=\mu_{f}, \\
& \mu_{\alpha}=\mu_{0}
\end{aligned}
$$

where $\mu_{f}$ is the magnetic permeability of poles and $\mu_{0}$ is the magnetic permeability of air.

In case of the quadrature-axis armature reaction, field lines are directed generally parallel to the $r$-axis within the air spaces between poles and generally perpendicular to the $r$-axis within the poles. Then the components of the magnetic permeability must be

$$
\begin{aligned}
& \mu_{r}=\mu_{0}, \\
& \mu_{\alpha}=\mu_{f} .
\end{aligned}
$$

In the anisotropic zone, Maxwell's equations determine electromagnetic field:

$$
\begin{aligned}
& \operatorname{rot} \bar{H}=0, \\
& \bar{B}=\operatorname{rot} \bar{A}
\end{aligned}
$$

In case of the cylindrical coordinate system, Maxwell's equations (4) are equivalent to the partial differential equation for the vector potential:

$$
\frac{1}{\mu_{\alpha}} \frac{\partial^{2} A}{\partial r^{2}}+\frac{1}{\mu_{\alpha} r} \frac{\partial A}{\partial r}+\frac{1}{\mu_{r} r^{2}} \frac{\partial^{2} A}{\partial \alpha^{2}}=0
$$

where $A$ is the $z$-component of the vector potential.

When the rotor revolves, we can see the traveling waves of the field. Therefore, the complex plane representation of the vector potential can be used. For the each $k$-th harmonic, the (5) turns into

$$
\frac{\partial^{2} \dot{A}_{k}}{\partial r^{2}}+\frac{1}{r} \frac{\partial \dot{A}_{k}}{\partial r}-\frac{\lambda_{k}^{2}}{r^{2}} \dot{A}_{k}=0
$$

where $\quad \lambda_{k}=k p \sqrt{\frac{\mu_{\alpha}}{\mu_{r}}}$.

The general solution of the equation (6) is

$$
\dot{A}_{k}=C_{1} r^{\lambda_{k}}+C_{2} r^{-\lambda_{k}} .
$$

The tangential component of the magnetic intensity (excluding the exciting field source) is

$$
\dot{H}_{\alpha k}=-\frac{1}{\mu_{\alpha}} \frac{\dot{A}_{k}}{\partial r}=-\frac{\lambda_{k}}{\mu_{\alpha} r}\left[C_{1} r^{\lambda_{k}}-C_{2} r^{-\lambda_{k}}\right] .
$$

Suppose that at the boundaries of the anisotropic zone, the vector potential and the tangential component of the magnetic intensity vector are known. As in Figure 1 for the first field harmonic, if $r=r_{1}$,

$$
\begin{aligned}
& \dot{A}_{1 k}=C_{1} r_{1}^{\lambda_{k}}+C_{2} r_{1}^{-\lambda_{k}}, \\
& \dot{H}_{1 k} r_{1}=-\frac{\lambda_{k}}{\mu_{\alpha}}\left[C_{1} r_{1}^{\lambda_{k}}-C_{2} r_{1}^{-\lambda_{k}}\right] .
\end{aligned}
$$

If $r=r_{2}$ (see Figure 1),

$$
\dot{A}_{2 k}=C_{1} r_{2}^{\lambda_{k}}+C_{2} r_{2}^{-\lambda_{k}},
$$




$$
\dot{H}_{2 k} r_{2}=-\frac{\lambda_{k}}{\mu_{\alpha}}\left[C_{1} r_{2}^{\lambda_{k}}-C_{2} r_{2}^{-\lambda_{k}}\right]
$$

Suppose that, for the first field harmonic, the equivalent $A$ - $H$-circuit (in which vector magnetic potential is considered equivalent to the voltage and the tangential component of the magnetic intensity vector multiplied by a radius is considered equivalent to the current) corresponds to the rotor as shown in Figure 2.

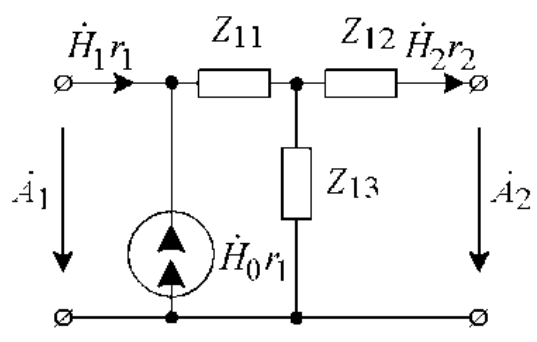

Figure 2. The equivalent $A$ - $H$-circuit of the rotor

The ideal current source $\dot{H}_{0} r_{1}$ corresponds to the first harmonic of the current expression (1):

$$
\dot{H}_{0} r_{1}=j \frac{\dot{I}_{0} \sin p \beta}{p \Theta \beta} .
$$

The expressions (9)-(12) describe the operation conditions of the equivalent $A$ - $H$-circuit. On the other hand, when operation conditions change, the constants of the equivalent $A-H$-circuit (including the impedances $Z_{11}, Z_{12}, Z_{13}$ ) shall not change. Therefore, we can determine the impedances $Z_{11}, Z_{12}, Z_{13}$ by analyzing the expressions (9)-(12) in the short-circuit conditions and the no-load conditions:

$$
\begin{aligned}
& Z_{11}=Z_{12}=\frac{\mu_{\alpha}}{\lambda_{1}} \frac{\left(\frac{r_{2}}{r_{1}}\right)^{\lambda_{1}}+\left(\frac{r_{1}}{r_{2}}\right)^{\lambda_{1}}-2}{\left(\frac{r_{2}}{r_{1}}\right)^{\lambda_{1}}-\left(\frac{r_{1}}{r_{2}}\right)^{\lambda_{1}}}, \\
& Z_{13}=\frac{\mu_{\alpha}}{\lambda_{1}} \frac{2}{\left(\frac{r_{2}}{r_{1}}\right)^{\lambda_{1}}-\left(\frac{r_{1}}{r_{2}}\right)^{\lambda_{1}}} .
\end{aligned}
$$

\section{THE CASCADE EQUIVALENT $\boldsymbol{A}$ - $H$-CIRCUIT OF THE GENERATOR}

The cascade equivalent $A-H$-circuit of the generator has two cells as shown in Figure 3 . The first cell $\left(Z_{11}, Z_{12}, Z_{13}\right)$ corresponds to the rotor and the second cell $\left(Z_{21}, Z_{22}, Z_{23}\right)$ corresponds to the air gap. Constants of the second cell may be deduced from expressions (14) and (15) when $\mu_{r}=\mu_{\alpha}=\mu_{0}$. For the first field harmonic,

$$
Z_{21}=Z_{22}=\frac{\mu_{0}}{p} \frac{\left(\frac{r_{3}}{r_{2}}\right)^{p}+\left(\frac{r_{2}}{r_{3}}\right)^{p}-2}{\left(\frac{r_{3}}{r_{2}}\right)^{p}-\left(\frac{r_{2}}{r_{3}}\right)^{p}}
$$




$$
Z_{23}=\frac{\mu_{0}}{p} \frac{2}{\left(\frac{r_{3}}{r_{2}}\right)^{p}-\left(\frac{r_{2}}{r_{3}}\right)^{p}}
$$

The air gap irregularity can be taken into account if the air gap is multiplied by

$$
\frac{k_{\delta}}{\alpha_{\delta}}
$$

where $\alpha_{\delta}$ is the pole overlap factor and $k_{\delta}$ is Carter's factor.

If the magnetic permeability of stator teeth is infinite then the zone of stator teeth may be removed from the $A-H$-circuit and the ideal current source $\dot{I}_{2}$ (that corresponds to the armature reaction) will be connected to the output of the second cell. In compliance with Ampere's circuital low, this current source is determined as

$$
\dot{I}_{2}=\dot{\delta} h \frac{b_{1}+b_{2}}{4 \pi} z_{2}
$$

where

$\dot{\delta}$ is the current density in the stator slots (the amplitude of the first harmonic);

$z_{2}$ is the number of stator teeth;

$h$ is the tooth height;

$b_{1}$ and $b_{2}$ are the widths of the slot (on the air gap and on the stator yoke).

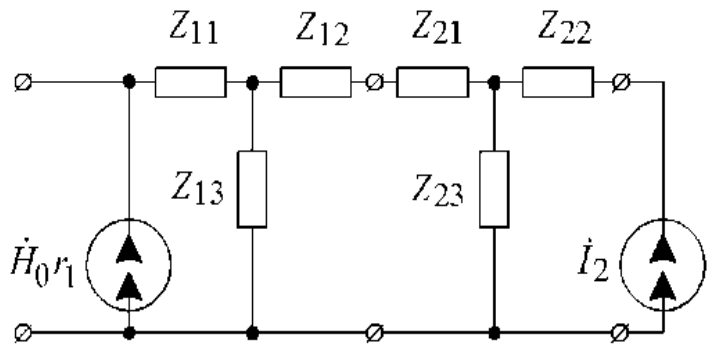

Figure 3. The cascade equivalent $A-H$-circuit of the generator

\section{RESULTS AND ANALYSIS}

Table 1 contains the initial data of the control calculations. The results of control calculations are offered in Figure 4 (the normal component of the magnetic induction vector on the stator). Both the numerical simulation and the calculations of the $A$ - $H$-circuit lead to the same result. It is evidence of the correctness of modeling.

Table 1. The initial data of the control calculations

\begin{tabular}{cccc}
\hline Parameter & Value & Parameter & Value \\
\hline The number of poles & 8 & Carter's factor & 1.22 \\
The pole relative permeability & 500 & The pole overlap factor & 0.73 \\
The rotor diameter & $632 \mathrm{~mm}$ & The exciting current & $4582.5 \mathrm{~A}$ \\
The pole height & $83.4 \mathrm{~mm}$ & The current density in the & $2 \mathrm{~A} / \mathrm{mm}^{2}$ \\
The air gap & $4 \mathrm{~mm}$ & stator slots & $\pi / 8$ \\
The number of stator teeth & 96 & $\Theta$ & $\beta$ \\
The tooth height & $53 \mathrm{~mm}$ & & $\pi / 16$ \\
The slot width (on the air gap) & $11.11 \mathrm{~mm}$ & & \\
The slot width (on the yoke) & $14.57 \mathrm{~mm}$ & & \\
\hline
\end{tabular}




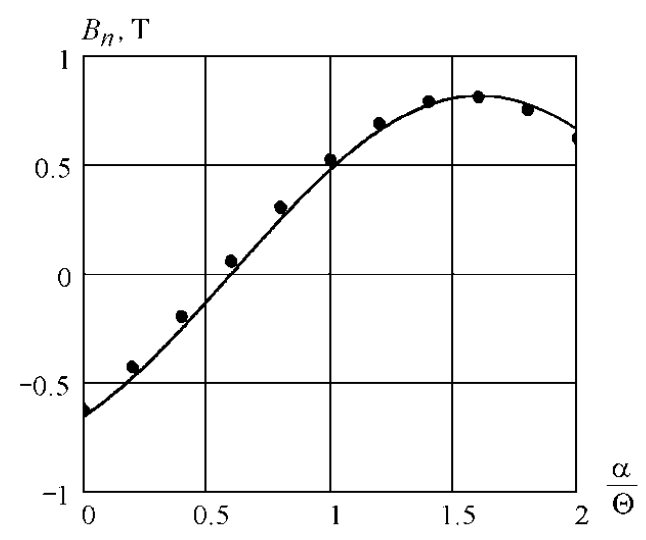

Figure 4. The magnetic induction vector on the stator (the normal component, the first field harmonic, the quadrature-axis armature reaction): the solid line is the cascade equivalent $A$ - $H$-circuit; points are the numerical simulation (ELCUT 5.1)

\section{CONCLUSION}

In this paper, the cascade equivalent $A$ - $H$-circuit of the synchronous salient-pole generator is considered. In this model, both the poles of the rotor and air spaces between the poles are replaced by the anisotropic zone. It makes it possible to avoid piecewise continuous Sturm-Liouville eigenfunctions. The constants of the $A$-H-circuit change depending on the field configuration (the exciting field or the longitudinal armature reaction or the quadrature-axis armature reaction). The air gap irregularity can be taken into account if the air gap is increased. Control calculations of the cascade equivalent $A$ - $H$-circuit and the numerical simulation lead to the same result. It is evidence of the correctness of modeling.

\section{REFERENCES}

[1] E. Mishkin, "Theory of the squirrel-cage induction motor derived directly from Maxwell's field equations," Quarterly Journal of Mechanics and Applied Mathematics, vol. 7, no. 4, pp. 472-487, 1954.

[2] S. A. Nasar, "Electromagnetic theory of electrical machines," Proceedings of the Institution of Electrical Engineers, vol. 111, no. 6, pp. 1123-1131, 1964.

[3] J. Greig and E. M. Freeman, "Traveling wave problem in electrical machine," Proceedings of the Institution of Electrical Engineers, vol. 114, no. 11, pp. 1681-1683, 1967.

[4] S. Williamson, "The anisotropic layer theory of induction machines and induction devices," IMA Journal of Applied Mathematics, vol. 17, no. 1, pp. 69-84, 1976.

[5] V. M. Kazansky and A. I. Inkin, "An electromagnetic model and elements of the induction machine's theory (in Russian)," Induction Micro-Machines. The Inter-University Conference on Induction Machines: Proc. (Kaunas), 1969, pp. 217-229.

[6] V. M. Kazansky, et al., "The analytical study of electromagnetic field in an active volume of the n-phase asynchronous machine with a non-slot stator (in Russian)," Induction electric motors with an allocated active stator layer: Proc., Novosibirsk: NETI, no. 2, pp. 41-57, 1972.

[7] A. I. Inkin and V. M. Kazansky, "Equivalent transformations of active zones in rotating electric machines (in Russian)," Electricity, vol. 1, pp. 42-45, 1975.

[8] E. M. Freeman, "Travelling waves in induction machines: input impedance and equivalent circuits," Proceedings of the Institution of Electrical Engineers, vol. 115, no. 12, pp. 1772-1776, 1968.

[9] E. M. Freeman, "Equivalent circuits from electromagnetic theory low-frequency induction devices," Proceedings of the Institution of Electrical Engineers, vol. 121, no. 10, pp. 1117-1121, 1974.

[10] E. M. Freeman and T. G. Bland, "Equivalent circuit of concentric cylindrical conductors in an axial alternating magnetic field," Proceedings of the Institution of Electrical Engineers, vol. 123, no. 2, pp. 149-152, 1976.

[11] A. I. Inkin, "The circuit approximation of linear mediums influenced by electromagnetic field (in Russian)," Electricity, no. 4, pp. 64-67, 1975.

[12] A. I. Inkin and B. V. Litvinov, "The synthesis of cascade equivalent circuits of induction electric machines on the basis of standard E-H-four-terminal networks (in Russian)," Electrotechnics, vol. 1, pp. 29-33, 1977.

[13] A. I. Inkin and Yu. G. Buchholz, "Foundations of the synthesizing of non-linear cascade equivalent circuits of an electric machine (in Russian)," Electricity, vol. 6, pp. 33-37, 1979.

[14] A. I. Inkin, "Electromagnetic fields and parameters of electric machines: tutorial (in Russian)," Novosibirsk: UKEA, pp. 1-464, 2002.

[15] B. V. Litvinov and O. B. Davidenko, "Standard cells and cascade equivalent circuits of electric machines: monograph (in Russian),” Novosibirsk: NSTU, pp. 1-215, 2008. 
[16] B. V. Litvinov and O. B. Davidenko, "The equivalent circuit of the synchronous reactive motor subject to higher spatial harmonic of magnetomotive force (in Russian)," Electricity, vol. 5, pp. 48-52, 2010.

[17] B. V. Litvinov and O. B. Davidenko, "Synchronous reactive motor with reduced magnetic conductance along transverse axis (in Russian)," Electrotechnics, vol. 3, pp. 15-20, 2010.

[18] L. Qaseer, et al., "Combined field and circuit theories in squirrel-cage induction motors based on micro-T circuit model," Aces Journal, vol. 26, no. 7, pp. 551-560, 2011.

[19] L. Qaseer, et al., "Closed-form analysis of squirrel-cage induction motors with anisotropic modeling of stator and rotor," IEEE Transactions on Energy Conversion, vol. 27, no. 3, pp. 553-560, 2012.

[20] V. V. Korneev, "Design coefficients and added losses of synchronous machines with permanent magnets and fractional tooth windings: candidate's dissertation: speciality 05.09.01 (in Russian)," Novosibirsk: NSTU, pp. 1-144, 2018.

[21] A. I. Inkin, et al., "The calculation of transformer induction heating system (in Russian)," Electrotechnics, vol. 11, pp. 34-37, 2000.

[22] F. N. Sarapulov, et al., "Mathematic models of linear induction machines on the basis of equivalent circuits 2nd edition (in Russian)," Yekaterinburg: UGTU-UPI, pp. 1-431, 2005.

[23] L. Qaseer, "Analysis of double and single sided induction heating systems by layer theory approach," Journal of electromagnetic analysis and applications, vol. 2, no. 7, pp. 403-410, 2010.

[24] L. Qaseer, "Micro-T circuit model for double and single sided induction heating systems," Applied computational electromagnetics society journal, vol. 25, no. 8, pp. 713-721, 2010.

[25] L. Qaseer, "Micro-T circuit model for the analysis of cylindrical induction heating systems," IEEE Transactions on energy conversion, vol. 25, no. 4, pp. 1021-1027, 2010.

[26] A. V. Blanc, "Electromagnetic field and eddy currents in the mantle of the ore-smelting furnace," IOP Conference Series: Materials Engineering, vol. 560, no. 1, 2019, pp. 1-7.

[27] A. V. Blanc, "The cascade equivalent A-H-circuit of the induction heating system with a superconducting inductor (in Russian)," Electrotechnics, vol. 2, pp. 43-49, 2020.

[28] A. I. Inkin, "The analytical treatment of magnetic field in the active volume of the electric machine with permanent magnets (in Russian)," Electricity, vol. 5, pp. 30-33, 1979.

[29] A. I. Inkin, "Analitical solution of magnetic field's equations in discrete structures of salient-pole electric machines (in Russian)," Electricity, vol. 8, pp. 18-21, 1979.

[30] A. I. Inkin, "The mathematical formulation of magnetic field in the volumes of salient-pole the electric machines," Electricity (in Russian), vol. 2, pp. 30-35, 1997.

[31] A. V. Blanc, "The analytical calculation of exciting field of synchronous machine on the base of the single piecewise continuous eigenfunction (in Russian)," Collected scientific papers of the NSTU, vol. 4, no. 38, pp. 3-8, 2004.

[32] A. I. Inkin and A. V. Blanc, "The approximate analytical calculation of exciting field of electric machines on the base of the piecewise continuous eigenfunction (in Russian)," Electricity, vol. 6, pp. 52-56, 2008.

[33] A. V. Blanc, "The A-H-equivalent circuit of a synchronous electric machine with tangential permanent magnets," Proceedings of the 2018 14th international scientific-technical conference on actual problems of electronic instrument engineering (APEIE-44894), vol. 1, no. 5, 2018, pp. 62-65.

[34] A. V. Blanc, "A cascade equivalent A-H-circuit of a permanent magnet excited electrical machine (in Russian)," Electricity, vol. 10, pp. 42-47, 2019.

[35] A. V. Blanc, "A cascade equivalent A-H-circuit of a synchronous salient-pole electric machine," IOP Conference Series: Materials Engineering, vol. 560, no. 1, 2019, pp. 1-7.

[36] A. Blanc, "Exciting field and quadrature-axis armature reaction in a cascade equivalent A-H-circuit of a salientpole generator," International Journal of Electrical and Computer Engineering (IJECE), vol. 10, no. 2, pp. 1674-1681, 2020.

\section{BIOGRAPHY OF AUTHOR}

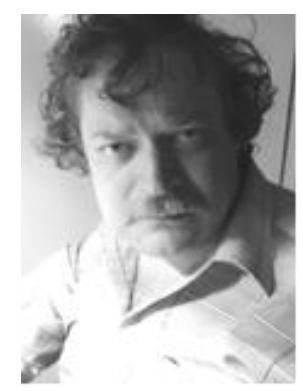

Alexey Valerievich Blanc was born in 1971. He received the Master's degree in Mechanical \& Electrical Engineering (in 1999) and the Candidate's degree in Engineering (in 2005) from the Novosibirsk State Technical University. Since 2005, he is the associate professor in the Department of the Theoretical Electrical Engineering in the Novosibirsk State Technical University. He has 60 scientific publications. His scientific works have to do with the engineering electrodynamics and the heat-transfer theory. 